\title{
Detecting Vehicle Motion using Deep Gaussian Mixture Model with SCI-kit Learn
}

\author{
Tamilarasu Viswanathan, N. Vinothkumar
}

\begin{abstract}
Detecting vehicle motions are a progressively significant part in road surveillance and Traffic organizing systems. This paper presents a new Deep Gaussian based mixture model that predicts accurate in detecting vehicle motions. Although the existing arrangements based on conventional Gaussian mixture model which is limited in insufficient of many distinct points which eliminate covariance and solutions relative to infinite likelihood. In the proposed scheme, the deep learning neural network is used for including the more points with nested mixture models. To overcome the effects of adding more points the modification achieved in architecture development. The validation of proposed scheme is achieved with real-time videos and process with scikit learn based model.
\end{abstract}

Keywords - motion detection, deep Gaussian mixture model, neural network, computer vision and scikit learn

\section{INTRODUCTION}

Increasing in Vehicles, the amount of traffic getting cumulative in common among all the countries. Subsequently, the development in road monitoring system is important in traffic organizing systems. The purpose of detecting pattern of traffic formation in specific area is helpful in creating the database for self-learning systems. There are many statistical data available to understand the vehicle detection schemes but they are lack of so many practical errors. Hence the actual video base database development schemes are come into practical for enhancing the surveillance systems. The investigation of the subdivision of moving objects build on background removal techniques is proposed in [1]. Diverse surroundings modeling methods appropriate to graphical investigating methods presented. Every pixel is exhibited with Gaussian distribution make on an arrangement of discrete edges, and separation is then achieved by means of a twin differencing approach. The necessity of engaging a circumstantial perfect apprise approach in hysterical outside locations. In order to eliminate unnecessary segmented structures, shadow recognition and exclusion approaches have been frequently engaged [1-3]. Moreover, to hold incomplete and comprehensive blockings available in camera recorder records tributary. Obstruction recognition is implemented by means of a prolonged Filter that estimates the situation and the extent of object vaulting sections. The difference between predicted and restrained zones find to classify the type and extent of an obstruction. Traffic organizing schemes based on vehicle detection specifically developed for accident identification at highway junctions assess the

Revised Manuscript Received on 14 August, 2019.

TamilarasuViswanathan, Electrical and Electronics Engineering Kumaraguru College of Technology, Coimbatore, Tamilnadu, India(Email: viswanathan.t.eee@kct.ac.in)

N.Vinothkumar, Electrical and Electronics Engineering Kumaraguru College of Technology, Coimbatore, Tamilnadu, India(Email: vinothkumar.n.eee@kct.ac.in) vehicle speed, and to predict the vehicles collision [4-7]. The commonly used techniques for vehicle route identification, route arrangement is proposed in [7-9].

In this paper, a new deep Gaussian based method is presented for identifying the vehicle motion detection. More nested mixture models are included for considering more point for better detection using scikit learn. The arrangement of this paper is structured as follows. In Section II, the deep Gaussian mixture model for vehicle motion detection is explained. Section III pronounces the proposed deep Gaussian detection method is explained in compare with existing schemes. Explanation and implementation of the proposed scheme with real-time video data are discussed in Section IV. The conclusion and the future scope of the proposed method is offered in Section V.

\section{DEEP GAUSSIAN MIXTURE MODEL (DGMM)}

Multiple layers of Gaussian Mixture Models is an upfront generalized as DGMM as shown in Figure 1. The stack of multiple GMM-layers constructed on top of one another. Every DGMM forms a similar density function name as shallow GMM which is based on module blend with an exponential number. A new set of parameters are incorporated for classifying the images into appropriate variations. This interpretation is achieved through selection proper layers in the architectures which categorize input real-time data set as change in basic properties line color, brightness, contrast and hues. Instead going of traditional movements' combination, here the variations are achieved through the proper selection of exponential number.

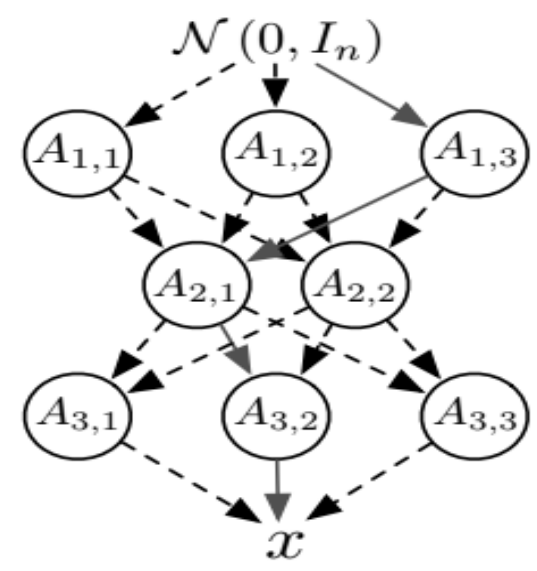

Fig.1. General Structue of Deep Gaussian Mixture Model (DGMM) 
The distribution of several calculation components easily identified through proper probability and the expansion stages or deep learning, with only limited communication between compute layers. The multivariate distribution model is developed for achieving the motion vehicle detection.

\section{PROPOSED DETECTING VEHICLE MOTION}

In this work, DGMM achieved through multivariate normal distribution of existing Gaussian model. The general variable $y$ which is transformation from the image proper variable $x$. The linear classification is denoted as

$$
y=m x+c
$$

The probability distribution for any mixture model is represented in terms of intermediate matrices $A$ and extended as

$$
p(y)=p\left(x \mid c_{i}, A_{i} A_{i}{ }^{T}\right)
$$

Where $\mathrm{i}=1,2,3 \ldots$ (Individual image samples)

In this special case, probability end estimator is included in end level and each deformations is constructed through the image structure is useful. These estimator is capable of identifying image alterations like over exposure, physical changes while capturing and other practical shortages. The overall block diagram of the proposed scheme is presented in Figure 2.

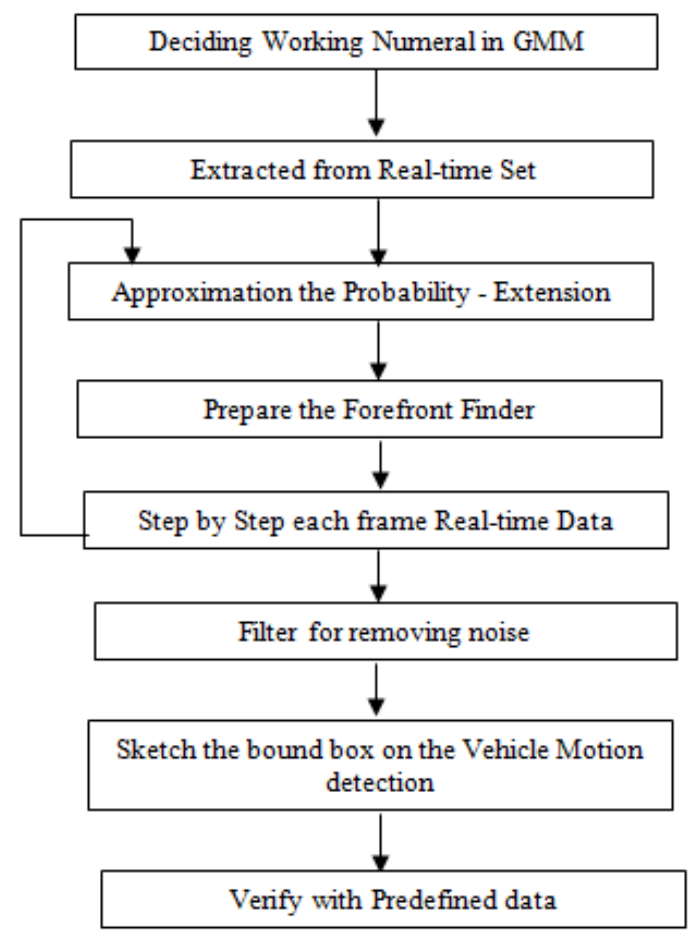

Figure 2:Overview of Proposed Deep Gaussian DGMM based vehicle motion detection.

The analysis of DGMM based vehicle motion detection to separate the individual process into a tracing and make the proper cataloging of vehicles through moving camera for better analysis. Here the objective the work begins with realtime motion detection and process the video frame into possible informative numeral in DGMM based neural network with multiple layers. The extracted numerals are placed into a weights of neural network. Next to approximation the Probability - Extension of the extracted images which is based on the (2). The automatic intermediate neural network transformation matrices are preloaded into the probability density with the size of $219 \times$ 219 matrices. At the end of the physical fitting, the indicator produces the consistent separation output from the probability existing function. The extracted figures and further steps after the segmentation from the GMM are shown in Figure 3, 4 and 5.

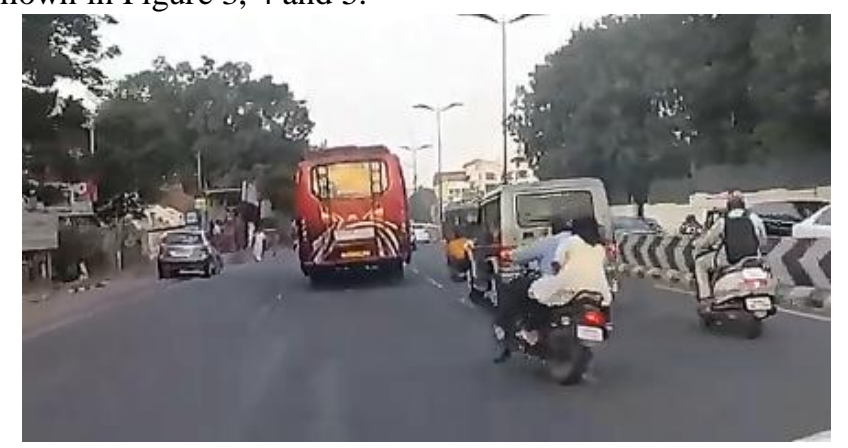

$$
\begin{aligned}
& \text { Figure 3.Extracted Realtime output taken from the } \\
& \text { Camera }
\end{aligned}
$$

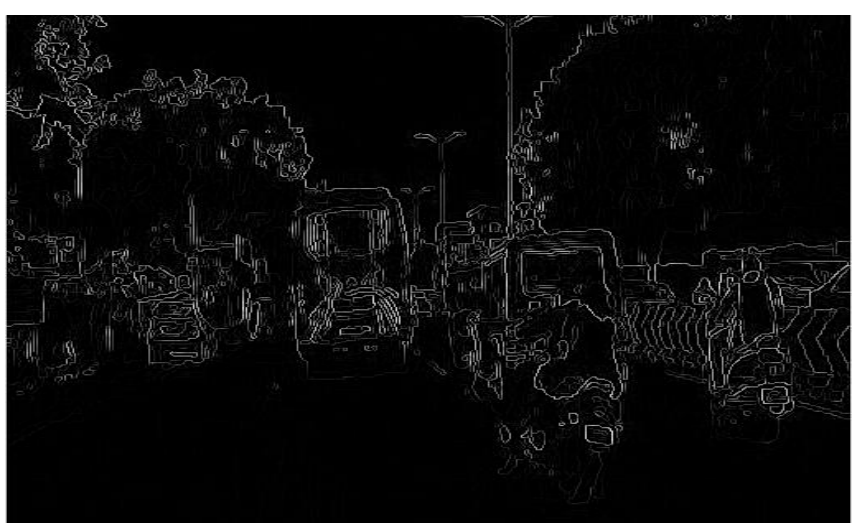

Figure 4.Prepare the forefront finder from approximate the probability and extension

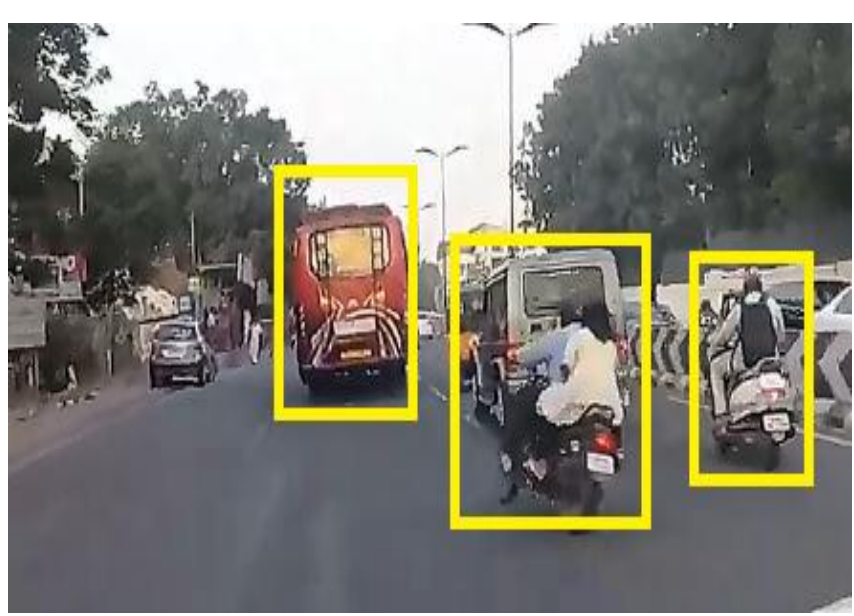

Figure 5.Findout the boundbox on the vehicle motion detection and crosscheck with predefined data

The forefront segmentation development is not accurate and comprises unwelcome scatter shown in Figure 4. In the last phase, the procedure continute for remaining video edges until the matched with accurate.

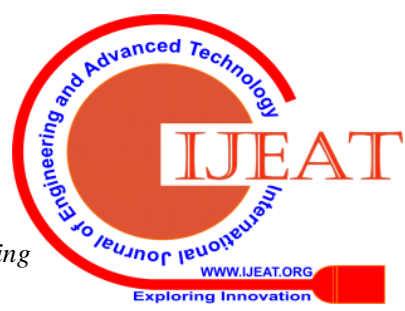




\section{DISCUSSION\& RESULTS}

The same extracted data is used under DGMM is compare with Deterministic model, Conventional neural network, structured Gaussian Model, K- Nearest neighbor algorithm, GMM, Modified GMM. Throughout the analysis, the transformation matrices size is taken as same and number segmentation cluster is same and categorized as two wheelers, three wheelers and Four Wheelers (Large Vehicles except Cars). The same number of groups is taken into account for all the mixture based techniques for doing better comparison. From the Table I, the random index which indicating the probability dissimilarity of different mixture models and misclassification of trained images. The conventional neural network evasion the reduced misclassification value of 0.012 for two wheelers and less value among the other schemes because of the less points are consider for the analysis. But the randomization index is very high which is not preferred. On the other hand, the Modified GMM value with Random index with high misclassification value which is not acceptable. Hence combining the advantages of this two and Proposed Deep GMM method with Random index and misclassification of $(0.889,0.301)$ for two wheelers, $(0.904,0.431)$ for three wheelers and $(0.771,0.522)$ for four wheels is needed for better vehicle detection.

\section{CONCLUSION}

\section{REFERENCES}

1. D. Gutchess, M. Trajkovics, E. Cohen-Solal, D. Lyons, and A. K. Jain, "A background model initialization algorithm for video surveillance," in Proc. 8th IEEE Int. Conf. Comput. Vis., Vancouver, BC, Canada, Jul. 2001, pp. 734-740.

2. W. Hu, T. Tan, L. Wang, and S. Maybank, "A survey on visual surveillance of object motion and behaviors," IEEE Trans. Syst., Man, Cybern. C, Appl. Rev., vol. 34, no. 3, pp. 334-352, Aug. 2004.

A. Prati, I. Mikic, C. Grana, and M. M. Trivedi, "Shadow detection algorithms for traffic flow analysis: A comparative study," in Proc. IEEE Intell. Transp. Syst. Conf., Oakland, CA, Aug. 2001, pp. 340-345

4. N. Vinothkumar,.V.Kumar Chinnaiyan, Pradish.M and Prabhakar karthikeyan, "Recommend cascade structure multilevel inverter with reduced power electronic components", Electric Power Components and Systems, Taylor and Francis publications 0(0), 1-6. DOI.doi.org/10.1080/15325008.2018.1496370.

5. N. Vinothkumar,.V.Kumar Chinnaiyan, Pradish.M and Prabhakar karthikeyan, "Multilevel Inverter Structure using Single Source and Double Source Module to Reduce Power Electronics Components", IET The Journal of Engineering, pp. 1-10, March 2017 (DOI:dx.doi.org/10.1049/joe.2017.0068).

6. Vinothkumar N, Viswanathan T, and Mathan kumar M, Intelligent street light controller with security system, IJITEE, 2S, 2018, 1-6

7. Vinothkumar N, Sequential Quadratic Programming (SQP) Based Selective Harmonic Elimination for Multilevel Inverter, IJITEE, 2S, 2018, 94-98.

8. N. Vinothkumar, V.Kumar Chinnaiyan and Pradish.M "Comparative analysis of SHE techniques for seven and nine level inverter with CSA, PSO and SA algorithms" Journal of electrical engineering (Communicated)
9. N. Vinothkumar,V.KumarChinnaiyan,Pradish.M and Prabhakar karthikeyan, "Simulated Annealing Based Selective Harmonic Elimination for Multi-level Inverter", Elsiver energy procedia, 17C, pp. 790 796,2017 